\title{
Endothelial trans-differentiation in glioblastoma recurring after radiotherapy
}

\author{
Ivana De Pascalis ${ }^{1}$ - Liliana Morgante ${ }^{2}$ - Simone Pacioni ${ }^{1}$ - Quintino Giorgio D'Alessandris ${ }^{1}$ - Stefano Giannetti ${ }^{2}$. \\ Maurizio Martini ${ }^{3} \cdot$ Lucia Ricci-Vitiani $^{4} \cdot$ Matteo Malinverno $^{5} \cdot$ Elisabetta Dejana ${ }^{5}$. Luigi M. Larocca $\mathbb{B}^{3}$. \\ Roberto Pallini ${ }^{1}$
}

Received: 11 May 2017 / Revised: 3 February 2018 / Accepted: 4 February 2018 / Published online: 30 April 2018

(c) United States \& Canadian Academy of Pathology 2018

\begin{abstract}
We hypothesized that in glioblastoma recurring after radiotherapy, a condition whereby the brain endothelium undergoes radiation-induced senescence, tumor cells with endothelial phenotype may be relevant for tumor neovascularization. Matched glioblastoma samples obtained at primary surgery and at surgery for tumor recurrence after radiotherapy, all expressing epidermal growth factor receptor variant III (EGFRvIII), were assessed by a technique that combines fluorescent in situ hybridization (FISH) for the EGFR/CEP7 chromosomal probe with immunostaining for endothelial cells (CD31) and activated pericytes ( $\alpha$ Smooth Muscle Actin). Five EGFRvIII-expressing paired primary/recurrent glioblastoma samples, in which the tumor cells showed EGFR/CEP7 amplification, were then assessed by CD31 and $\alpha$ Smooth Muscle Actin immunofluorescence. In glomeruloid bodies, the ratio between CD31+ cells with amplified EGFR/CEP7 signal and the total CD31+ cells was $0.23 \pm 0.09$ (mean \pm sem) and $0.63 \pm 0.07$ in primary tumors and in recurrent ones, respectively $(p<0.002$, Student- $t$ test). In capillaries, the ratio of CD31+ cells with amplified $E G F R / C E P 7$ over the total CD31+ cells lining the capillary lumen was $0.21 \pm 0.06$ (mean \pm sem) and $0.42 \pm 0.07$ at primary surgery and at recurrence, respectively $(p<0.005$, Student- $t$ test). Expression of $\alpha$ Smooth Muscle Actin by cells with EGFR/CEP7 amplification was not observed. Then, in glioblastoma recurring after radiotherapy, where the brain endothelium suffers from radiation-induced cell senescence, tumor-derived endothelium plays a role in neo-vascularization.
\end{abstract}

These authors contributed equally: Ivana De Pascalis, Liliana Morgante and Simone Pacioni.

These authors jointly supervised this work: Luigi M. Larocca, Roberto Pallini.

Electronic supplementary material The online version of this article (https://doi.org/10.1038/s41379-018-0046-2) contains supplementary material, which is available to authorized users.

$\triangle$ Roberto Pallini

roberto.pallini@unicatt.it

1 Institute of Neurosurgery, Università Cattolica del Sacro Cuore, Rome, Italy

2 Institute of Human Anatomy, Università Cattolica del Sacro Cuore, Rome, Italy

3 Institute of Pathology, Università Cattolica del Sacro Cuore, Rome, Italy

4 Department of Oncology and Molecular Medicine, Istituto Superiore di Sanità, Rome, Italy

5 New Strategies to Inhibit Tumor Angiogenesis Program Fondazione Italiana per la Ricerca sul Cancro, Institute of Molecular Oncology Fondazione, Milan, Italy

\section{Introduction}

Glioblastoma is a highly angiogenic malignancy. In this tumor, several cellular mechanisms contribute to new vessel formation, that include, 1) sprouting from pre-existing vessels (neo-angiogenesis); 2) differentiation of circulating bone marrow-derived cells into endothelial progenitors (vasculogenesis); 3) generation of functional vessel-like networks lined by tumor cells (vascular mimicry); and 4) endothelial/pericyte trans-differentiation. The last mechanism, which postulates that glioma stem-like cells may acquire vascular phenotypes and directly contribute to tumor vasculature, has recently been identified based on in vitro and in vivo studies [1-5]. However, the actual impact of endothelial/pericyte trans-differentiation under clinical conditions remains to be determined [2]. It is known that the brain endothelium proliferates at high rates while building the vascular tree of glioblastoma [6], and that radiotherapy inhibits sprouting and proliferation of the brain endothelium, which in turn is much more resistant to 
temozolomide $[7,8]$. We hypothesized that tumor recurring after radio-chemotherapy, where neo-angiogenesis is impaired due to radiation-induced damage of the brain endothelium, represents a clinical condition in which glioblastoma may adopt the endothelial trans-differentiation as a supportive mechanism to build up its vasculature.

\section{Materials and methods}

\section{Endothelial cell cultures}

Human endothelial cells (HMVECs; Bio-Wittaker, Walkersville, MD) were maintained in complete endothelial cell growth medium (EGM-2; Bio-Wittaker) containing endothelial cell basal medium (EBM-2; Bio-Wittaker) supplemented with endothelial cell Bullet kit (Bio-Wittaker; 2\% FBS, hEGF-2, hVEGF, R3-IGF-1, ascorbic acid, hydrocortisone, heparin, gentamicin and amphotericin-B). Cells were grown at $37{ }^{\circ} \mathrm{C}$ in a humidified atmosphere of $5 \%$ $\mathrm{CO}_{2}-95 \%$ air. Cells were used between passage 2 and 5 , plated at a density of $2.5 \times 10^{4}$ cells $/ \mathrm{mL}$, in triplicate, in 96well plates. For evaluation of radiation, endothelial cells were exposed to single doses of acute cesium-137 (137Cs) gamma irradiation (10 and $50 \mathrm{~Gy}$; dose rate $0.8 \mathrm{~Gy} / \mathrm{min}$ ).

\section{Radiation of mouse brain}

Experiments on animals were approved by the Ethical Committee of the Università Cattolica del Sacro Cuore, Rome, Italy. CD1 mice (male, 25-30 g; Charles River, Milan, Italy) were anesthetized with intraperitoneal injection of diazepam $(2 \mathrm{mg} / 100 \mathrm{~g})$ followed by intramuscular injection of ketamine $(4 \mathrm{mg} / 100 \mathrm{~g})$. Animal skulls were immobilized in a custom-made head holder with a lead mask covering both eyes and nose and then exposed to $6 \mathrm{~Gy}$ dose of 137Cs gamma irradiation at $0.8 \mathrm{~Gy} / \mathrm{min}$ rate. Radiation was administered twice weekly over 2 weeks (total dose 24 Gy). Two weeks later, the mice were deeply anesthetized and transcardially perfused with $0.1 \mathrm{M}$ PBS ( $\mathrm{pH}$ 7.4) then treated with $4 \%$ paraformaldehyde in $0.1 \mathrm{M}$ PBS. The brain was removed, stored in $30 \%$ sucrose buffer overnight at $4{ }^{\circ} \mathrm{C}$, and serially cryotomed at $20 \mu \mathrm{m}$ on the coronal plane.

\section{Immunohistochemistry and senescence-associated $\beta$-galactosidase activity assay}

Expression of $\gamma$-H2A.X, a component of the DNA damage response in senescent cells [9], was assessed in endothelial cells using a primary antibody against y-H2A.X (S139; rabbit monoclonal, 1:250, Cell Signaling, Herts, UK). Cultured endothelial cells and brain sections were assessed for senescence-associated $\beta$-galactosidase activity, a widely accepted marker for cellular senescence, according to the manufacturer's instructions (\#KAA0022RF, Millipore, Temecula, CA). For CD31 immunostaining of brain sections, slices were incubated with rabbit polyclonal antiCD31 (1:50; Santa Cruz Biotechnology, Segrate, Milan, Italy). The reaction was developed using the avidin-biotin peroxidase complex (ABC method, Vector Laboratories, Burlingame, CA) and 3,3'-diamidinobenzidine (Sigma, St. Louis, MO) as a chromogen.

\section{Clinical material}

From the databases of the Institutes of Neurosurgery and Pathology, Università Cattolica del Sacro Cuore, we retrieved 10 adult patients showing the following, 1) they underwent craniotomy for resection of histologically confirmed glioblastoma (WHO IV) in the supratentorial compartment; 2) they underwent reoperation for local tumor recurrence; 3) fractionated focal external beam radiation therapy (60 Gy) was completed before surgery for tumor recurrence, and 4) epidermal growth factor receptor variant III (EGFRvIII) was expressed both by primary tumor and by recurrent one (Table 1) [10]. All neurosurgical procedures were performed at Università Cattolica del Sacro Cuore. Patients were aged 29 to 69 years at the time of primary surgery (median age, 51.5 years) and included 2 men and 8 women. After primary surgery, all patients received radiotherapy to limited fields ( $2 \mathrm{~Gy}$ per fraction once daily 5 days a week; 60 Gy total dose) and temozolomide according to the Stupp protocol [11]. Patients were candidate for reoperation whether the Karnofsky performance status was $\geq 70$ and the recurrent tumor for site and extension was amenable to gross total removal (Supplementary Fig. S1). All patients provided written informed consent according to the research proposals approved by the Ethical Committee of Università Cattolica del Sacro Cuore.

\section{Combination of immunophenotyping and fluorescence in situ hybridization (FISH)}

Immunofluorescence was performed on deparaffinized sections as described elsewhere [3]. Sections were incubated with primary antibody against the endothelial cell marker anti-human CD31 (Monoclonal Mouse, clone JC70A, 1:50 dilution, Dako, Milan, Italy) or the pericyte cell marker anti-human $\alpha$ Smooth Muscle Actin (Monoclonal Mouse, clone 1A4. 1:100 dilution, Dako). Pericytes of histologically normal human brain microvessels either lack $\alpha$ Smooth Muscle Actin staining or express this marker at low levels [12, 13], however, tissues with microvascular proliferation, such as glioma, consistently show pericyte staining for $\alpha$ Smooth Muscle Actin [14, 15]. After immunostaining, FISH analysis was performed using LSI 
Table 1 Summary of clinical and pathological data

\begin{tabular}{|c|c|c|c|c|c|c|c|}
\hline \multirow[t]{2}{*}{ Case \# } & \multirow[t]{2}{*}{ Age(years)/sex } & \multirow[t]{2}{*}{ Tumor location } & \multirow[t]{2}{*}{ Overall survival (mos) } & \multicolumn{2}{|c|}{ Primary surgery } & \multicolumn{2}{|c|}{ Recurrence } \\
\hline & & & & EGFRvIII & $E G F R$ & EGFRvIII & $E G F R$ \\
\hline 1 & $64 / \mathrm{F}$ & Occipital & 17 & Pos & Amp & Pos & Amp \\
\hline 2 & $52 / \mathrm{F}$ & Temporal & 35 & Pos & Amp & Pos & Amp \\
\hline 3 & $50 / \mathrm{F}$ & Parietal & 31 & Pos & Amp & Pos & Amp \\
\hline 4 & $47 / \mathrm{M}$ & Temporal & 42 & Pos & Amp & Pos & Amp \\
\hline 5 & $69 / \mathrm{F}$ & Frontal & 5 & Pos & Amp & Pos & Amp \\
\hline 6 & $52 / \mathrm{M}$ & Temporal & 8 & Pos & Pol & Pos & Pol \\
\hline 7 & $29 / \mathrm{F}$ & Frontal & 52 & Pos & Pol & Pos & Pol \\
\hline 8 & $61 / \mathrm{F}$ & Parietal & 20 & Pos & Pol & Pos & Pol \\
\hline 9 & $51 / \mathrm{F}$ & Parietal & 26 & Pos & Pol & Pos & Pol \\
\hline 10 & $47 / F$ & Temporal & 32 & Pos & Pol & Pos & Pol \\
\hline
\end{tabular}

EGFR epidermal growth factor receptor, EGFRvIII epidermal growth factor receptor variant III, Amp amplified, Pol polysomic, Pos positive

EGFR Probe (Vysis EGFR/CEP7 FISH Probe Kit, Abbott, Rome, Italy). The ratio between the number of CD31+ cells with amplified EGFR/CEP7 signal and the total number of CD31+ cells was assessed both in glomeruloid bodies and in capillaries of glioblastoma at primary surgery and at surgery for recurrence. In each tumor specimen, at least 40 glomeruloid bodies and 40 capillaries were analyzed. In our material, capillaries were identified as single-layered or multi-layered microvascular structures characterized by hyperplasia and hypertrophy of CD31-expressing endothelial cells in the vessel wall. Glomeruloid bodies were defined as tuft-like microvascular structures characterized by multilayered endothelial cells together with $\alpha$ Smooth Muscle Actin expressing pericytes. Though we did not use anti-CD34 imunostaining, our definitions of capillaries and glomeruliod bodies closely approach the microvascular sprouting and glomeruloid vascular proliferations, respectively, described by Birner et al. [16].

\section{Double immunofluorescence}

Surgical specimens were fixed in $4 \%$ paraformaldehyde at $4{ }^{\circ} \mathrm{C}$ for 3 days, cryoprotected in $30 \%$ sucrose in PB at $4{ }^{\circ} \mathrm{C}$ for 4 days, and cut on a cryostate into $40 \mu \mathrm{m}$ thick sections. Following an antigen-retrieval step (sections were boiled for $30 \mathrm{~min}$ in $10 \mathrm{mM}$ citric acid, $0.05 \%$ Tween 20 , pH 6.0, in water bath at $95-100{ }^{\circ} \mathrm{C}$ ), the sections were incubated overnight at $4{ }^{\circ} \mathrm{C}$ in $\mathrm{PB}$ with $0.3 \%$ Triton $\mathrm{X}-1000,0.1 \%$ normal donkey serum with primary rabbit anti-human ERG (1:50; Dako Agilent, Santa Clara, CA) and rat anti-antiCollapsin Response Mediated Protein 5 (CRMP5, 1:50, Millipore, Billerica, MA). Slices were then rinsed in PB and incubated with Alexa Fluor 647-conjugated donkey antirabbit, Alexa Fluor 488-conjugated donkey anti-rat and Alexa Fluor 555-conjugated donkey anti-goat (1:400; Thermo Fisher Scientific, Waltham, MA) secondary antibodies for $2 \mathrm{~h}$ at room temperature. Following steps of washing in PB and incubation with DAPI (1:4000; SigmaAldrich, St. Louis, MO) the sections were stained with $0.3 \%$ Sudan Black B (Sigma Aldrich)—to reduce autofluorescence-and coverslipped before images acquisition with a laser confocal microscope (Leica SP5).

\section{Statistical analysis}

Student's $t$-test was used to analyze data using Statistica (version 5.5; Statsoft) software.

\section{Results}

Radiation experiments on cultured endothelial cells and on mouse brain were set up in order to ascertain that $\gamma$-H2A.X immunohistochemistry and senescence-associated $\beta$-galactosidase assay could reliably detect the radiation-induced senescence of the brain vascular endothelium, which was postulated to trigger the endothelial trans-differentiation of glioblastoma recurring after radiotherapy. In cultured endothelial cells, the expression of $\gamma$-H2A.X was much higher after radiation (Supplementary Fig. S2, left). Under baseline conditions, $4-6 \%$ of the endothelial cell population showed senescence-associated $\beta$-galactosidase activity that increased to 27 and to $35 \%$ by $96 \mathrm{~h}$ after exposure to 10 and $50 \mathrm{~Gy}$ radiation, respectively (Supplementary Fig. S2, left). In vivo, the brain endothelium of control untreated mice does not show cell senescence, as demonstrated by senescenceassociated $\beta$-galactosidase assay coupled with anti-CD31 immunohistochemistry (Supplementary Fig. S2, right). Conversely, after brain radiation ( $24 \mathrm{~Gy}$ in $6 \mathrm{~Gy}$ fractions) the brain vascular endothelium showed clear signs of cell senescence. When applied to our clinical material, these methods demonstrated that in tumors recurring after 
Fig. 1 Assessment of radiationinduced endothelial cell damage in glioblastoma recurring after radiotherapy. H\&E staining (a, d), double immunostaining with anti-CD31 antibody (red) and anti- $\gamma$-H2A.X antibody (green) $(\mathbf{b}, \mathbf{e})$, and senescenceassociated $\beta$-galactosidase activity (blue) (c, f) in glioblastoma at primary surgery (a-c) and at surgery for tumor recurrence after radiation therapy (d-f). After radiotherapy, several cells of the human brain endothelium undergo nuclear cell damage. The arrows point out senescent endothelial cells. Scale bars, $30 \mu \mathrm{m}$
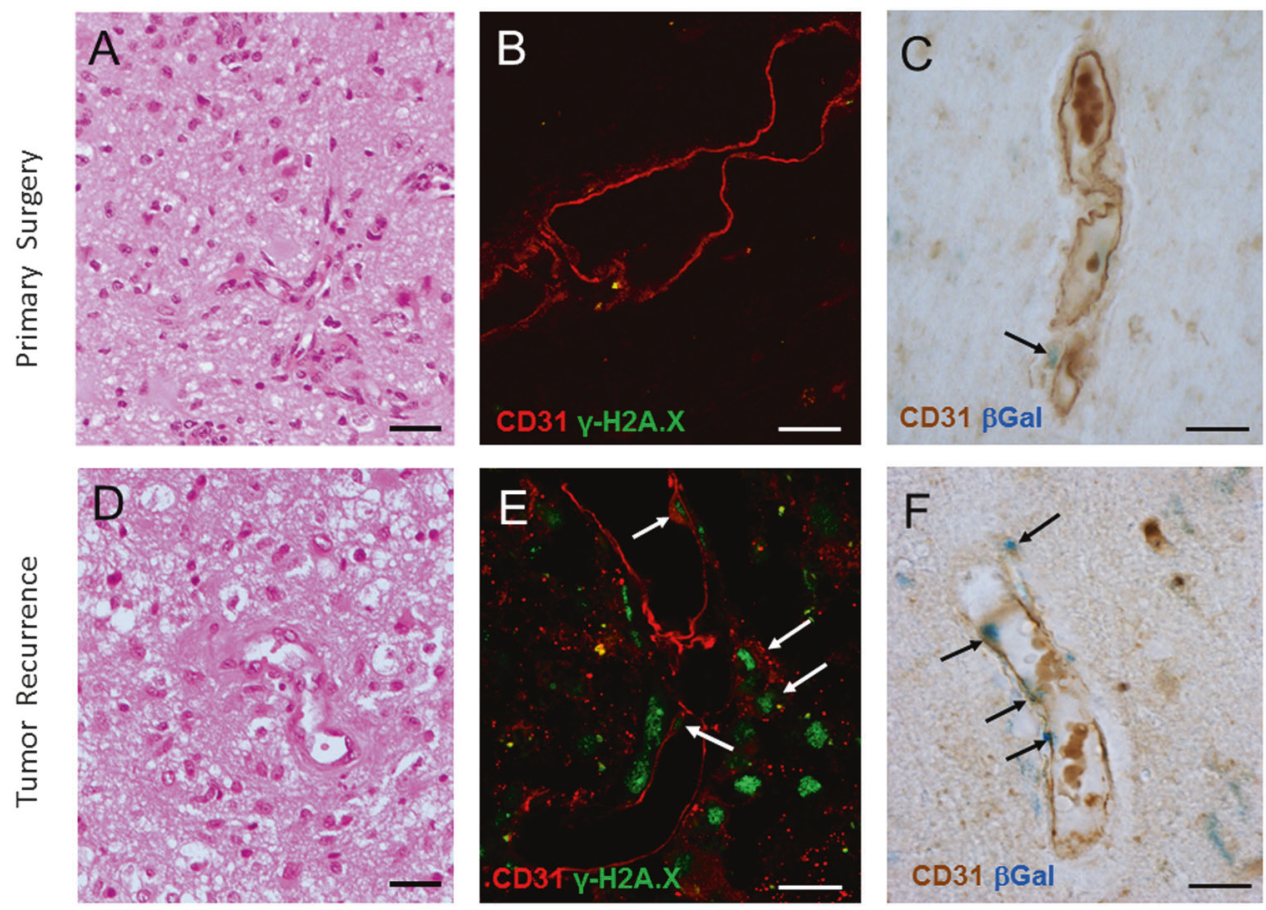

radiotherapy a substantial fraction of the vascular endothelium suffers nuclear damage and cell senescence (Fig. 1). On histological examination, the recurrent tumors showed features of radiation-induced vasculopathy, such as telangiectatic and hyalinized vessels [17]. Endothelial hyperplasia was commonly seen, resulting in enlarged and multilayered microvessels (Supplementary Fig. S3).

FISH analysis revealed that out of 10 paired primary/ recurrent glioblastoma with EGFRvIII expression, 5 of them showed amplified $E G F R / C E P 7$ gene, whereas an additional 5 cases had polysomy of EGFR/CEP7 (Fig. 2 and Table 1). Combining FISH for tumor-specific chromosomal probes with anti-CD31 immunofluorescence allows recognizing the relationships between the tumor cells and endothelium, as well as the endothelial phenotype of tumor cells [3]. However, given that combining immunostaining with FISH detaches probes from chromosomes and this may confuse analysis of polysomic nuclei, only specimens with amplified EGFR/ CEP7 signals were analyzed. Glomeruloid bodies of glioblastoma at primary surgery were mainly composed by endothelial cells with diploid EGFR/CEP7 signals, demonstrating their origin from the brain endothelium (Fig. 3a). Cells with amplified $E G F R / C E P 7$ showing CD31 expression were occasionally incorporated into the glomerulus. In radiated glioblastoma, however, several CD31 expressing cells with amplified $E G F R / C E P 7$ populated the glomeruloid bodies (Fig. 3a). The ratio between CD31+ cells with amplified $E G F R / C E P 7$ and the total CD31+ cells was $0.23 \pm 0.09$ (mean \pm sem) and $0.63 \pm 0.07$ in primary tumors and in recurrent ones, respectively $(p<0.002$, Student $-t$ test).
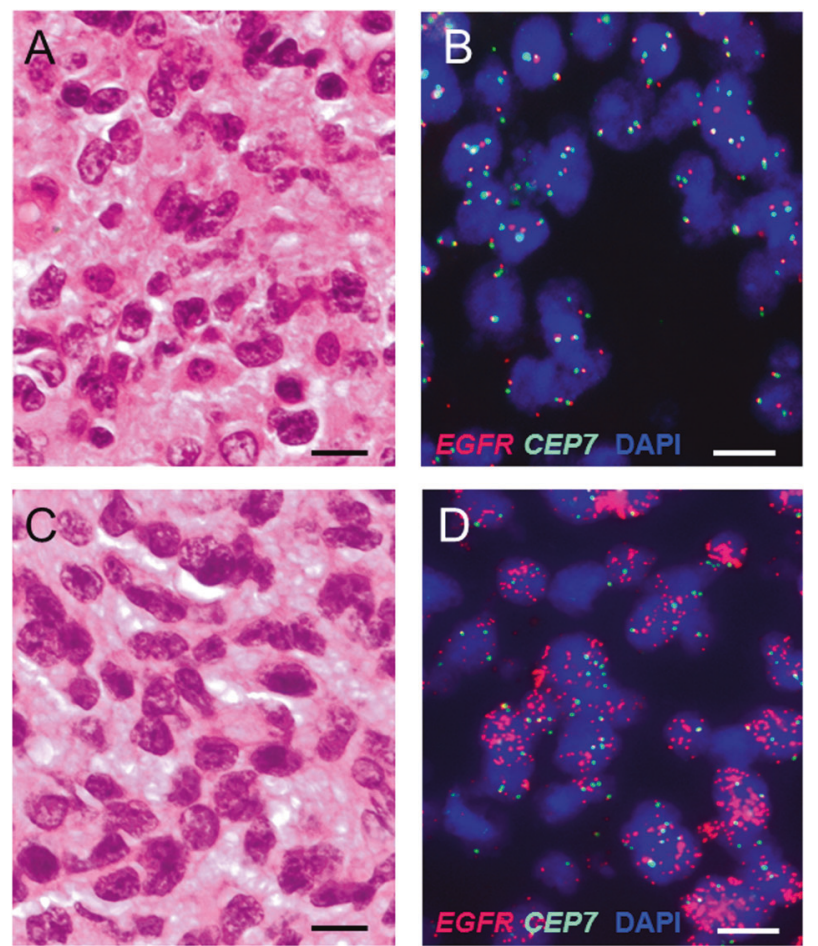

Fig. 2 Fluorescence in situ hybridization (FISH) of glioblastoma tumors. $\mathrm{H} \& \mathrm{E}$ staining $(\mathbf{a}, \mathbf{c})$ and FISH analysis using the $E G F R / C E P 7$ chromosomal probe in two EGFRvIII expressing recurrent tumors showing polysomy (b) or amplification (d) of the EGFR/CEP7 gene. Cell nuclei are counterstained with DAPI. Scale bars, $10 \mu \mathrm{m}$

In capillaries of glioblastoma at primary surgery, the tumor cells were often found to line the vessel lumen (Fig. 3a). The majority of these cells did not express the endothelial marker 
A
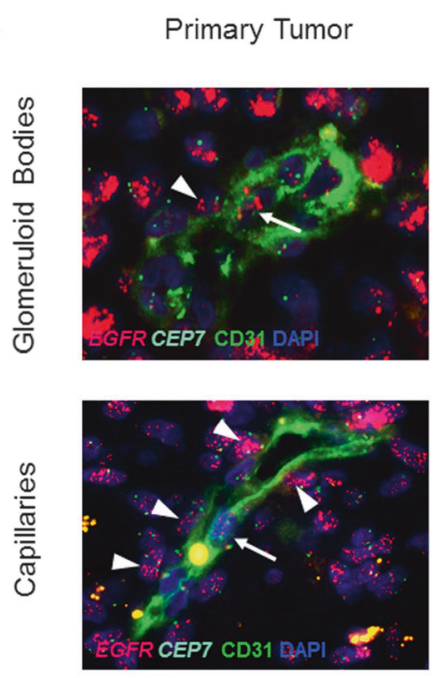

Recurrence
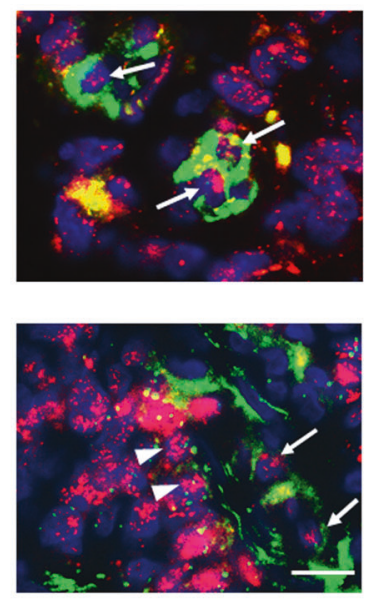

B

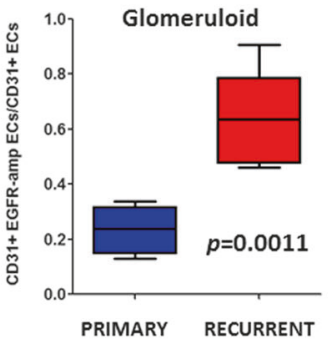

C

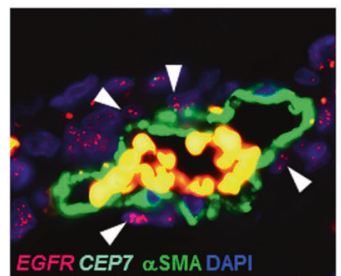

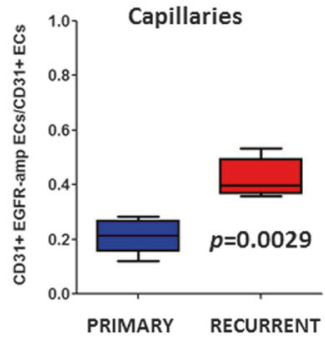

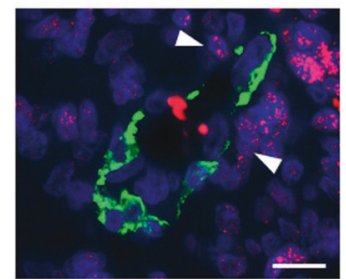

Fig. 3 Combined immunofluorescence for endothelial/pericyte markers and FISH on glioblastoma at primary surgery and at recurrence. FISH using the EGFR/CEP7 chromosomal probe (a) in EGFRvIII expressing glioblastoma showing EGFR/CEP7 amplification (EGFR, red; CEP7, blue marine) combined with anti-CD31 endothelial immunostaining (green). Capillaries and glomeruloid bodies of two glioblastomas before (Primary Tumor) and after radiotherapy (Recurrence) are presented. In tumors at primary surgery, the majority of tumor cells with amplified $E G F R / C E P 7$ surrounding the glomerulus or lining the capillary lumen do not express CD31 (arrowheads). The arrows point out $\mathrm{CD} 31+$ cells with amplified EGFR/CEP7. In

CD31, suggesting vascular mimicry. Conversely, capillaries of recurrent glioblastoma showed several tumor cells, as identified by amplified EGFR/CEP7 signals, that showed expression of CD31 in their cytoplasm (Fig. 3a). The ratio of $\mathrm{CD} 31+$ cells with amplified $E G F R / C E P 7$ over the total $\mathrm{CD} 31+$ cells lining the capillary lumen was $0.21 \pm 0.06$ (mean \pm sem) and $0.42+0.07$ in tumors at primary surgery and in the recurrent ones, respectively $(p<0.005$, Student $-t$ test). However, given that we assessed capillaries characterized by hyperplasia of CD31-expressing endothelial cells and that in the context of glioblastoma not all capillaries show endothelial hyperplasia, it is likely that our analysis may have somewhat overestimated the phenomenon.

To detect the expression of the endothelial phenotype by the tumor cells, we also performed double immunofluorescence combining either the anti-ERG antibody or the anti-CD31, as endothelial cell markers, with the antiCRMP5 antibody, which has recently been demonstrated to selectively stain glioma cells [18-20], in a subset of matched specimens $(n, 3)$ obtained at primary surgery and at surgery for tumor recurrence after radio-chemotherapy. The anti-CRMP5 immunostaining was preliminarly validated on brain xenografts of patient-derived glioma stem-like cells that expressed the green fluorescent protein (GFP). In this material, CRMP5 selectively stained a fraction (about 10 percent) of tumor cells (Supplementary Fig. S4A). Specificity of the anti-CRMP5 immunostaining for the glioma recurrent tumors, both glomeruloid bodies and capillaries show several cells with amplified $E G F R / C E P 7$ that express CD31 in their cytoplasm (arrows). Graphs (b) showing quantitative assessment of CD31+ cells with amplified $E G F R / C E P 7$ in glomeruloid bodies and capillaries of glioblastoma at primary surgery and at surgery for tumor recurrence. FISH with $E G F R / C E P 7$ chromosomal probe (EGFR, red; CEP7, blue marine) combined with anti- $\alpha$ Smooth Muscle Actin immunostaining (green) (c). Cells with amplified EGFR/CEP7 that are in close relationships with tumor vascularity do not express the pericyte cell marker in their cytoplasm (arrowheads; left panel, primary tumor; right panel, recurrent tumor). Scale bars, $20 \mu \mathrm{m}$

cells was also assessed following irradiation using brain xenografts of patient-derived GFP-expressing glioma stemlike cells (Supplementary Fig. S4B).

In patients' glioblastoma at primary surgery, the CRMP5-expressing tumor cells did not show ERG immunostaining in their nuclei, which was observed only in the nuclei of endothelial cells (Supplementary Fig. S4C). Conversely, in glioblastoma recurring after radiochemotherapy about 7-8 percent of CRMP5-positive tumor cells expressed ERG (Supplementary Figs. S4C-D). Double anti-CRMP5 and anti-CD31 immunohistochemistry confirmed the aberrant endothelial phenotype of glioma cells following radiation (Supplementary Fig. S4E).

There was no expression of $\alpha$ Smooth Muscle Actin in the cytoplasm of cells with amplified EGFR/CEP7 signal both in tumors at primary surgery and in the recurrent ones (Fig. 3b), suggesting that pericytes of glioblastoma vasculature do not recognize a neoplastic origin.

\section{Discussion}

Recently, several studies have explored the phenomenon of tumor-derived vasculature in glioblastoma with discrepant, sometimes conflicting, results. [1-5, 21] Discrepancies concern the phenotype of tumor-derived vascularity (endothelial vs. pericyte) and the quantitative relevance of 
such phenomenon as well. In addition, the issue whether glioma cells with aberrant vascular phenotype may be anatomically, i.e., at the ultra-structural level, and/or functionally integrated in the vessel wall lining remains to be elucidated. Albeit adjuvant radio-chemotherapy substantially increases median survival of glioblastoma patients, it induces a series of events on the brain vasculature, like DNA damage and senescence of endothelial cells, that may influence tumor regrowth. Glioblastoma is likely able to adopt various strategies to build up its vasculature and neo-angiogenesis is commonly regarded as the main one. In glioblastoma recurring after radiation, however, neo-angiogenesis may lose efficiency due to the radiation-induced senescence of the brain endothelium.

The present study shows that in glioblastoma recurring after radiation therapy the brain endothelium undergoes cell senescence, and that the fraction of tumor cells expressing an endothelial phenotype increases up to 2-2.7 folds as compared with tumors at primary surgery. Therefore, in glioblastoma recurring after radiation therapy the tumor cellderived vascularity appears to play a much more relevant role. In this condition, the glioma stem-like cells are supposed to compensate the radiation-induced senescence of the brain endothelium and to favor neo-vascularization, which is of critical importance for the regrowth of glioblastoma. The apparent paradox of the sensitivity of the brain vasculature to radiation and the resistance of glioblastoma to radiotherapy can be explained postulating that, under certain environmental conditions, the fraction of glioblastoma cells with stem-like features may become able to express endothelial markers and to form vascular channels. Our findings may help explaining failure of anti-angiogenetic therapies in glioblastoma.

Acknowledgements This study was partly funded by Associazione Italiana per la Ricerca sul Cancro (IG 2013 N.14574 to RP and IG 2014 N.15584 to LR-V).

\section{Compliance with ethical standards}

Conflict of interest The authors declare that they have no conflict of interest.

\section{References}

1. Cheng L, Huang Z, Zhou W, et al. Glioblastoma stem cells generate vascular pericytes to support vessel function and tumor growth. Cell. 2013;153:139-52.

2. Hu B, Wang Q, Wang YA, et al. Epigenetic activation of WNT5A drives glioblastoma stem cell differentiation and invasive growth. Cell. 2016;167:1281-95.
3. Ricci-Vitiani L, Pallini R, Biffoni M, et al. Tumor vascularization via endothelial differentiation of glioblastoma stem-like cells. Nature. 2010;468:824-8.

4. Soda Y, Marumoto T, Friedmann-Morvinski D, et al. Transdifferentiation of glioblastoma cells into vascular endothelial cells. Proc Natl Acad Sci USA. 2011;108:4274-80.

5. Wang R, Chadalavada K, Wilshire J, et al. Glioblastoma stem-like cells give rise to tumour endothelium. Nature. 2010;468:829-33.

6. Pallini R, Pierconti F, Falchetti ML, et al. Evidence for telomerase involvement in the angiogenesis of astrocytic tumors: expression of human telomerase reverse transcriptase messenger RNA by vascular endothelial cells. J Neurosurg. 2001;94:961-71.

7. Kioi M, Vogel H, Schultz G, et al. Inhibition of vasculogenesis, but not angiogenesis, prevents the recurrence of glioblastoma after irradiation in mice. J Clin Invest. 2010;120:694-705.

8. Virrey JJ, Guan S, Li W, et al. Increased survivin expression confers chemoresistance to tumor-associated endothelial cells. Am J Pathol. 2008;173:575-85.

9. Wang C, Jurk D, Maddick M, et al. DNA damage response and cellular senescence in tissues of aging mice. Aging Cell. 2009;8:311-23.

10. Montano N, Cenci T, Martini M, et al. Expression of EGFRvIII in glioblastoma: prognostic significance revisited. Neoplasia. 2011;13:1113-21.

11. Stupp R, Mason WP, van den Bent MJ, et al. Radiotherapy plus concomitant and adjuvant temozolomide for glioblastoma. N Engl J Med. 2005;352:987-96.

12. Verbeek MM, Otte-Höller I, Wesseling P, et al. Induction of alpha-smooth muscle actin expression in cultured human brain pericytes by transforming growth factor-beta 1 . Am J Pathol. 1994;144:372-82.

13. Tigges U, Welser-Alves JV, Boroujerdi A, et al. A novel and simple method for culturing pericytes from mouse brain. Microvasc Res. 2012;84:74-80.

14. Sun H, Guo D, Su Y, et al. Hyperplasia of pericytes is one of the main characteristics of microvascular architecture in malignant glioma. PLoS One. 2014;9:e114246.

15. Louis DN, Suvà ML, Burger PC, et al. Glioblastoma, IDH-wild type. In: Louis DN, Ohgaki H, Wiestler OD, Cavenee WK, editors. WHO classification of tumours of the central nervous system. Revised. 4th edn. Lyon, France: IARC; 2016. pp. 28-45.

16. Birner P, Piribauer M, Fischer I, et al. Vascular patterns in glioblastoma influence clinical outcome and associate with variable expression of angiogenic proteins: evidence for distinct angiogenic subtypes. Brain Pathol. 2003;13:133-43.

17. Perry A, Schmidt RE. Cancer therapy-associated CNS neuropathology: an update and review of the literature. Acta Neuropathol. 2006;111:197-212.

18. Brot $\mathrm{S}$, Malleval $\mathrm{C}$, Benetollo $\mathrm{C}$, et al. Identification of a new CRMP5 isoform present in the nucleus of cancer cells and enhancing their proliferation. Exp Cell Res. 2013;319:588-99.

19. Tan F, Thiele CJ, Li Z. Collapsin response mediator proteins: potential diagnostic and prognostic biomarkers in cancers (Review). Oncol Lett. 2014;7:1333-40.

20. Moutal A, Honnorat J, Massoma P, et al. CRMP5 controls glioblastoma cell proliferation and survival through notch-dependent signaling. Cancer Res. 2015;75:3519-28.

21. Rodriguez FJ, Orr BA, Ligon KL, et al. Neoplastic cells are a rare component in human glioblastoma microvasculature. Oncotarget. 2012;3:98-106. 